\title{
Vehicle Routing Problem with Simultaneous Delivery and Pick-up with an Improved Genetic Algorithm
}

\author{
Nuo Zhu* \\ Information center of CAAC, 155\# Dongsi Xidajie, Beijing, 100710, China \\ ${ }^{*}$ Corresponding author
}

\begin{abstract}
Based on the traditional theory and algorithms of vehicle routing problem, the multi-objective VRPSDP model is established in considering the minimum of the number of vehicles and the transportation costs. The genetic algorithm is used as the solving algorithm of the model in this paper, in order to ensure the effectiveness of the chromosomes in the iterative process, the chromosome encoding and the genetic arithmetic operators, which are more suitable for solving the problem of the vehicle operating route are devised. Simulation experiments are done in the application of the modified Solomon R101 case, the optimal objective function value and the optimal program of arranging the vehicle routes are respectively obtained by the basic GA and the improved GA. According to the comparative analysis the convergence process of the optimal solution on two genetic algorithms, it is validated that the model is reasonable and the solving algorithm is effective.
\end{abstract}

Keywords—vehicle routing problem; genetic algorithm; pick-up and delivery; transportation cost

\section{INTRODUCTION}

The VRPSDP was proposed by Min [1], which solved the problem of sending and returning books between one central library and 22 local libraries. Gendreau et a1 [2] researched on VRPSDP of having one vehicle only. Dethloff J [3] worked on the VRPSDP from the aspect of converse logistics. Tang and Galvão [4] also proposed an alternative mathematical formulation for VRPSDP. Bianchessi and Righini [5] compared the performance of various constructive, local search and TS algorithms designed for the VRPSPD, whereas Zachariadis et al. [6] propose a hybrid metaheuristic approach based on TS controlled by a guiding mechanism for diversifying the conducted search. Finally the most recent articles on the VRPSPD have been published by Gajpal and Abad [7] and Ai and Kachitvichyanukul [8]. The former work proposes an Ant Colony System methodology which employs a construction rule as well as two multi-route local search schemes, whereas the latter paper presents a Particle Swarm Optimization (PSO) algorithm. A practical VRPSPD variant has been tackled by Privé et al. [9] who have considered a problem which arises from the distribution of soft drinks and collection of recyclable containers. In this paper, the improved VRPSDP model is set up on considering the impact of the amount of vehicles and the total cost of transportation. The model which based on basic genetic algorithm and modified genetic algorithm is worked out by Matlab program. In order to ensure the effectiveness of the chromosomes in the iterative process, chromosome encoding method of genetic algorithm and genetic operators are devised which are more suitable for solving vehicle routing problem. Simulation experiments are done by using emendatory Solomon R101 illustrative example, the optimal vehicle routing programs and the optimal objective function values are got by two algorithms, the results are compared according to the convergence process of the optimal solution. The validity of the VRPSDP model and the feasibility of the modified algorithm are proved in this paper.

\section{MODEL DESCRIPTION}

Most of the existing VRPSDP models are aimed to minimize the total distance of vehicles, or minimize the overall cost of operating. However, the outlay of starting vehicles plays an important role in the whole cost of transportation. The more amount of vehicles that joins in distributing goods, the higher cost of transportation. Therefore, this paper proposes a modified model to minimize the total cost which considering the running costs of vehicles and the starting costs of vehicles.

$$
\begin{aligned}
& \min F(i, j, k)=\alpha \sum_{i=0}^{n} \sum_{j=0}^{n} \sum_{k=1}^{m} c_{i j} x_{i j k}+\beta \sum_{k=1}^{m} \sum_{j=1}^{n} x_{0 j k} \\
& \text { s.t. }\left\{\begin{array}{l}
\sum_{k=1 i=0}^{m} \sum_{i j k}^{n} x_{i j k}=1 \\
\sum_{j=1}^{n} y_{0 j k}=\sum_{i=0}^{n} \sum_{j=1}^{n} d_{i} x_{i j k} \\
\sum_{i=1}^{n} y_{i 0 k}=\sum_{i=0}^{n} \sum_{i j k}^{n} p_{i} x_{i j k} \\
\left.\sum_{j=1}^{n} y_{0 j k} \leq Q p_{i}-d_{i j k}\right) \sum_{i=0}^{n} x_{i j k}=\sum_{i=0}^{n} y_{j i k} \\
\sum_{i=1}^{n} y_{i 0 k} \leq Q x_{i j k} \\
0 \leq y_{i j k} \leq Q x_{i j k} \\
p_{i} \geq 0, d_{i} \geq 0, Q \geq 0, i, j \in R, k \in V, i \neq j
\end{array}\right.
\end{aligned}
$$

$R$ - the set of node, $R=\{i\}$, i=0 as a distribution center, $i=1,2, \ldots, n$ as a client node; $V$ - the set of vehicles, $V=\{k\}, k=1,2, \ldots, m ; \alpha$-the transportation cost of unit 
distance; $\beta$-the cost of per vehicle start-up; $Q_{\text {- the load }}$ capacity of vehicle; $C$ - the distance between each node, $C=\left\{c_{i j}\right\}, i, j \in \mathrm{R} ; d_{i}$ the amount of delivery goods to the client node i, $i \in R ; p_{i}$ — the amount of pick-up goods from the node $\mathrm{i} i \in R ; y_{i j k}$-the load weight of vehicle $\mathrm{k}$ running from node i to j directly;

$$
x_{i j k}=\left\{\begin{array}{l}
x_{i j k}=1 \text {, vehicle k serves node } i \text { and } j \text { directly } \\
x_{i j k}=0 \text {, otherwise }
\end{array}\right.
$$

To make practical VRPSDP abstracted into mathematical models, it's essential to establish the assumptions as follows: a) only an initial point which is a distribution center, each vehicle starts from the initial point and return to it after completing the task; b) the goods that are distributed and reclaimed can be mixed; c) the demands of each customer node are known; d) vehicles must provide services for all of the nodes; e) the load capacity of each vehicle is known; f) the distance between each client node, and the distance between the clients and distribution center are known; g) each client can only be served by one vehicle; h) each client can only be served once; i) each vehicle can only run along one route; j) pick-up and delivery goods can be completed simultaneously on the client nodes; k)the weight of an individual customer's goods can't exceed the load capacity of the vehicle.

\section{IMPROVED GENETIC ALGORITHM}

\section{A. Chromosome Coding}

Chromosome is done in the way of integral coding, which means each individual $i$ is a full array of the natural numbers from 1 to $\mathrm{n}$. In addition, each natural number corresponds to the number of client node along the route of the vehicle, and the order of natural numbers in each individual is the actual distribution order of the vehicle.

\section{B. Initializing Colony}

Since real-valued natural numbers are adapted as the way of encoding VRPSDP and the form of solution is based on the ranking, the initialized colony can generate a full array with popsize client nodes at any time and form a two-dimensional matrix of $n^{*}$ popsize. In the matrix, $n$ stands for the number of client nodes, popsize equals the amount of the colony.

\section{Fitness Value}

In the function, value(i) means the total transportation cost of the i-th individual. The fitness function fitness $(i)=1 /$ value $(i), i=1,2, \ldots$, popsize $\quad$ is adapted. It is necessary to calculate the total transportation distance.

\section{Selection of Operators}

The roulette of the non-back rounds on the basis of the taxis is adapted to select operator in this paper. On the assumption that the population size is $\mathrm{M}$, generate the parent colony called as $\mathrm{Z}$. The value of the fitness of each individual is $f\left(a_{i}\right)$, and the initial state of the offspring group is $X=\{\}$. Then the implementation process is described as follows:

a) calculate the fitness values of all the individuals and array the values in descending order $\left(Z^{\prime}\right)$. The greatest fitness of the individual in the parent colony called as $\mathrm{k}$ is recorded, $f\left(a_{k}\right)=\max \left(f\left(a_{1}\right), f\left(a_{2}\right), \ldots, f\left(a_{M}\right)\right)$;

b) calculate the summation of the fitness values of all the chromosomes in the colony $Z^{\prime}$;

c) calculate the probability that each chromosome will be selected, $P_{b_{i}}=f\left(b_{i}\right) / \sum_{i=1}^{M} f\left(b_{i}\right)$;

d) calculate the cumulative probability of each chromosome, which is recorded as $Q_{K}=\sum_{i=1}^{k} p_{i}$;

e) rotate the wheel, with generating $M$ random numbers between 0 and 1 which are defined as r. If $r \leq Q_{1}$, the chromosome $b_{1}$ will be selected; and if not, the k-th chromosome ${ }^{b} k(2 \leq k \leq M)$ will be selected, in that condition, $r$ can fit the constraint of $Q_{k-1} \leq r \leq Q_{k}$. The values of $\xi_{1}, \xi_{2}, \ldots, \xi_{M}$ in each interval are recorded, where $\xi_{i}$ stands for the number of random numbers in the region named i, $i=1,2, \ldots, M$;

f) The individual ${ }^{b}$ is corresponding to the region $\mathrm{j}$ where the maximum value of $\xi$ exists, $\xi_{j}=\max \left\{\xi_{1}, \xi_{2}, \ldots, \xi_{M}\right\}$, is selected as the individual ${ }^{M} j$ which is pitched on this round.

$$
\begin{aligned}
& \left\{\begin{array}{l}
M_{j}=b_{\min }\left(i_{1}, i_{2}, \ldots, i_{j},\right) \\
M_{j}=b_{j}, \text { else }
\end{array}\right. \\
& g)_{j} \text { is merged into } X,\left\{\begin{array}{l}
X(t)=X(t-1) \cup M_{j} \text { thus the } \\
X(0)=\varnothing
\end{array}\right.
\end{aligned}
$$
equation $X=\left\{c_{1}, c_{2}, \ldots c_{m}\right\}$ can be generated;

h) find out the individual with the lowest fitness value in the offspring group, then the parent individual $\mathrm{k}$ is taken into substitute for the offspring individual $\mathrm{j}$, $f\left(c_{i}\right)=\min \left(f\left(c_{1}\right), f\left(c_{2}\right), \ldots, f\left(c_{M}\right)\right)$;

i) store all the newly selected individuals and return to the new colony. 


\section{E. Intercrossing Arithmetic Operator}

The way of coding based on the natural numbers is adapted in this paper, so the design of the intercrossing arithmetic operators refer to the partially matched crossover method applying to the problem of the integer coding. Different from the traditional crossover arithmetic operator, this paper inserts the cross-section ahead of the first gene on the chromosome of the opposite party, then remove the genes which are the same ones between the part of the original individual and the gene of the cross-section. This kind of new improved intercrossing arithmetic operators can avoid the case that the solution isn't feasible in the form, and maintain the diversity of the colony in a certain extent, which can avoid falling into local optimal solution.

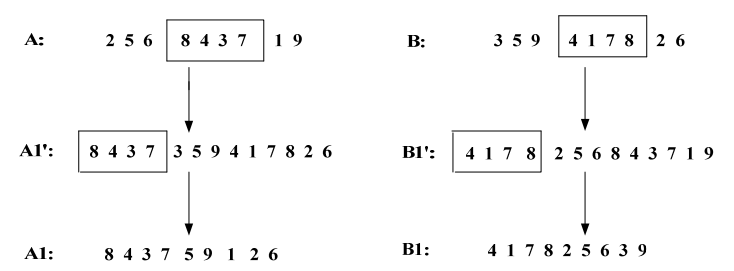

FIGURE I. SKETCH MAP OF THE INTERCROSS PROCESS

\section{F. Mutation Arithmetic Operator}

It is less likely that species change into mutations, so the main function of the mutation operator in the genetic algorithm is assisting the evolution. In this paper, the mutation manipulation takes the method of reverse the individual with coding the natural numbers. The colony randomly selects one individual at a certain mutation probability and generates two mutation points, then does reversal operation on the element in the middle of the mutation point.

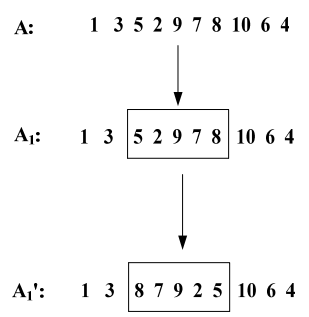

FIGURE II. SKETCH MAP OF THE VARIATION PROCESS

\section{SIMULATION EXPERIMENTS}

\section{A. Establishment of the Experiments}

In the example of Solomon R101, it is known that the number of the client nodes is 100 , the number of vehicles is 25 , and the maximum load weight of the vehicle is 100 . As the data of the demanded amount of the pick-up at the client nodes doesn't exist in the R101 case, which does not meet the actual situation of the VRPSDP. In this paper, the data of the amount of the pick-up at the client nodes is specially added on the basis of the R101 case, the method of generating data is mainly divided into two steps. To the selected nodes where pick-up goods and delivery goods can happen simultaneously, the pick-up goods quantity at the node is randomly generated, according to the scope of the demanded quantity of goods being delivered at the node and the maximum load weight of the vehicles.

TABLE I. TABLE OF PARAMETER

\begin{tabular}{cc}
\hline Parameter name & Value \\
\hline Chromosome length & $L=100$ \\
Colony size & $N=500$ \\
Maximum times of & Gen $=300$ \\
iterations & $p_{C}=0.85$ \\
Crossover probability & $p_{m}=0.01$ \\
Mutation probability & \\
\hline
\end{tabular}

\section{B. Result Analysis}

In order to get a more scientific and effective solution, the simulation experiments on improving the case of R101 are done for many times. Several results are got through the simulation experiments: the average transport distance is 1970.38, the average quantity of the vehicles to start is 17.2, the value of the average optimal objective function is 20563.8, and the average iteration times of getting the optimal objective function is 260. The optimal solution which got at the third experiment is the best, the objective function value is 20451, the optimal vehicle route is shown as Table II, and the optimal solution is shown as Figure III.

TABLE II. TABLE OF THE EXPERIMENT RESULT OF THE IMPROVED R101 INSTANCE BASED ON THE IMPROVED GA

\begin{tabular}{|c|c|c|c|c|}
\hline the optimal route & cars & distance & $\begin{array}{l}\text { objective } \\
\text { function }\end{array}$ & $\begin{array}{c}\text { iteration } \\
\text { times }\end{array}$ \\
\hline $0-83-92-100-98-7-18-0$ & \multirow{16}{*}{17} & \multirow{16}{*}{1960.1} & \multirow{16}{*}{20451} & \multirow{16}{*}{235} \\
\hline $0-96-5-84-82-1-0$ & & & & \\
\hline $0-39-4-41-22-56-12-0$ & & & & \\
\hline $0-42-94-37-86-45-0$ & & & & \\
\hline $0-50-20-71-30-33-26-0$ & & & & \\
\hline $0-27-78-66-35-34-54-0$ & & & & \\
\hline $0-81-65-32-31-0$ & & & & \\
\hline $0-36-49-64-90-93-87-6-0$ & & & & \\
\hline $\begin{array}{c}0-62-10-63-11-47-46-17-0 \\
0-16-85-44-43-57-0\end{array}$ & & & & \\
\hline $0-38-14-75-25-55-58-0$ & & & & \\
\hline $0-2-15-67-74-72-0$ & & & & \\
\hline $0-68-73-95-13-40-0$ & & & & \\
\hline $\begin{array}{c}0-69-70-51-80-24-29-77-9- \\
88-52-0\end{array}$ & & & & \\
\hline $0-28-97-59-21-23-0$ & & & & \\
\hline 0-48-19-8-60-61-91-99-0 & & & & \\
\hline 0-76-79-3-53-89-0 & & & & \\
\hline
\end{tabular}




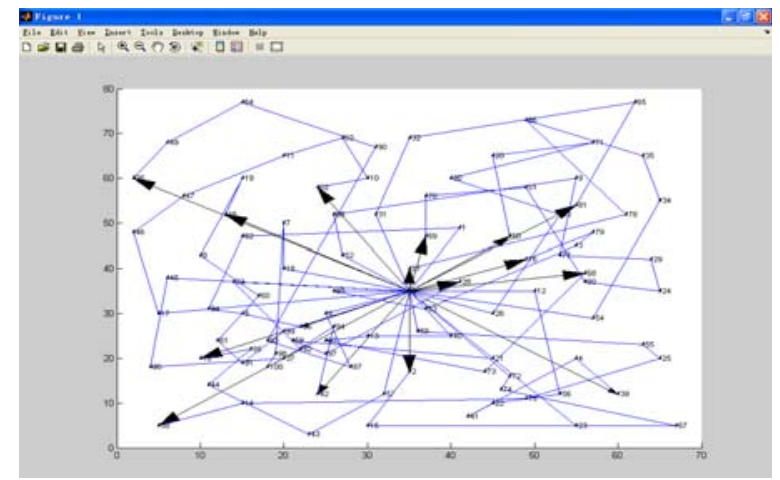

FIGURE III. THE OPTIMAL OF VEHICLE ROUTING BASED ON THE IMPROVED GA

In order to validate the fine nature of the improved genetic algorithm designed in this paper, the basic genetic algorithm is specially adapted on the same computer to solve the abovementioned example; the results of the two algorithms are compared and analyzed. To ensure the comparability of the algorithms, the parameters in the working process of the basic genetic algorithm should be consistent with the ones in the improved genetic algorithm. Under the same conditions, the optimal solution and the optimal route based on the basic genetic algorithm are respectively shown as Table III.

TABLE III. TABLE OF THE EXPERIMENT RESULT OF THE IMPROVED R101 INSTANCE BASED ON THE BASIC GA

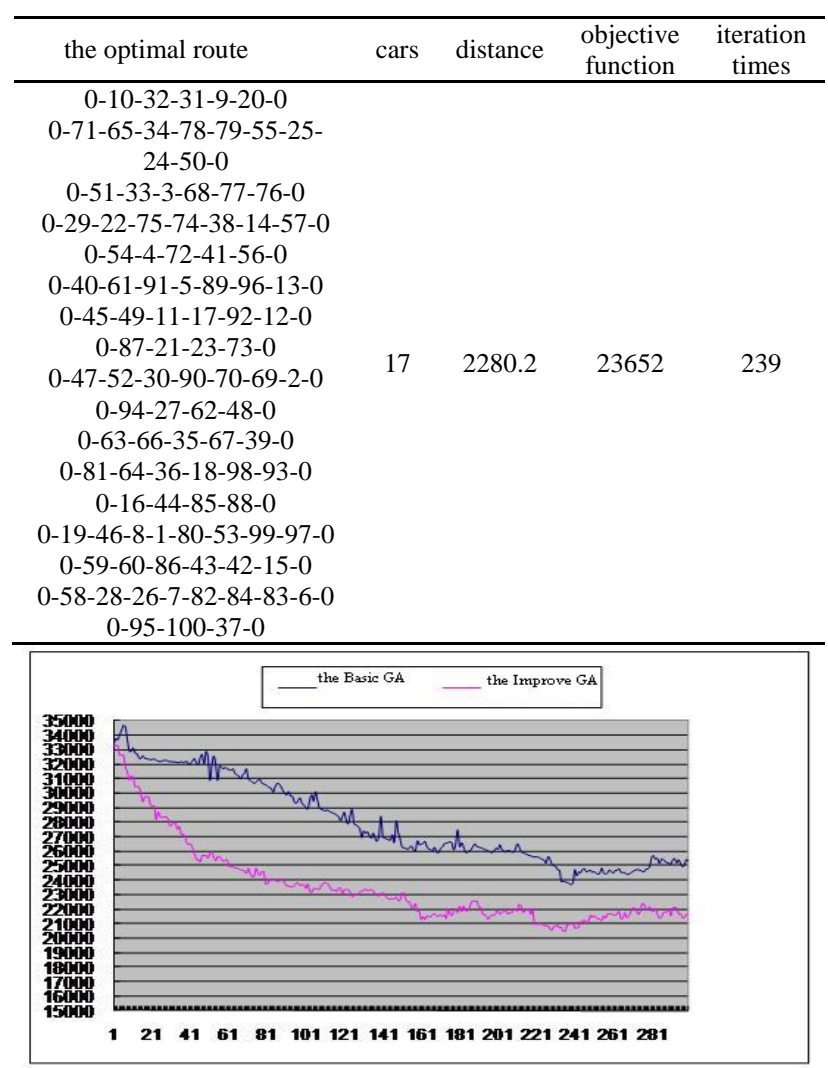

FIGURE IV. SKETCH MAP OF ITERATIVE PROCESS OF THE OPTIMAL SOLUTION BASED ON THE BASIC GA AND THE IMPROVED GA
The comparison of the convergence processes of the optimal solutions of the two algorithms is shown as Figure IV, the fluctuation of the basic GA based on the traditional roulette is relatively large, the convergence speed is slow, and the transformation begins to become relatively stable at the 233rd generation. In this paper, the multi-wheel roulette selecting operators based on the taxis has been improved on the algorithm performance, and the transformation tends to be relatively stable at the 155th generation, the convergence speed is faster than the basic roulette operator. In addition, the value of the optimal objective function obtained through the improved genetic algorithm is more ideal and fit for the need of low transport costs. Therefore, the improved genetic algorithm which has validity and feasibility performs better than the basic genetic algorithm.

\section{CONCLUSIONS}

The VRPSDP mathematical model aimed to obtain the minimum transportation cost is established based on the traditional theory of vehicle routing problem, the quantity of the vehicles and the total transportation cost are considered. The chromosome encoding and the genetic arithmetic operators that more suitable for solving the problem of the vehicle operating route are devised, which can ensure the effectiveness of the chromosomes in the iterative process. The solutions to the model based on the basic genetic algorithms and improved genetic algorithms are worked out through the Matlab program. The simulation experiments are done on the VRPSDP problem studied in this paper in the application of the modified Solomon R101 case, the programs of the optimal vehicle routes and the values of the optimal objective function are obtained on the two kinds of the genetic algorithms. The results are compared with each other according to the convergence process of the optimal solution, it is validated that the VRPSDP model and the improved algorithm in this paper are feasible and effective.

\section{ACKNOWLEDGMENT}

This paper is financially supported by the National Natural Science Foundation of China (Nos. 11422221 and 11672289).

\section{REFERENCES}

[1] Min H. The multiple vehicle routing problems with simultaneous delivery and pick-up points. Transportation Research. 1989. 23(5):377386.

[2] Gendreau M, Laporte G, Vigo D. Heuristics for the travelling salesman problem with pickup and delivery. Computers and Operations Research, 1999. 26(7):699-714.

[3] Dethloff J. Vehicle routing and reverse logistics: the vehicle routing problem with simultaneous delivery and pick-up. OR Spektrum, 2001. 23(1):79-96.

[4] Tang F A, Galvao R D. A tabu search algorithm for the vehicle routing problem with simultaneous pick-up and delivery service. Computer \& Operations Research. 2006.33(3):595-619.

[5] Bianchessi, N., Righini, G., Heuristic algorithms for the vehicle routing problem with simultaneous pick-up and delivery. Computers \& Operations Research. 2007. 34 (2), 578-594.

[6] Zachariadis, E.E., Tarantilis, C.D., Kiranoudis, C.T. A hybrid metaheuristic algorithm for the vehicle routing problem with simultaneous delivery and pick-up service. Expert Systems with Applications 2009. 36 (2), 1070-1081. 
[7] Gajpal, Y., Abad, P. An ant colony system (ACS) for vehicle routing problem with simultaneous delivery and pickup. Computers \& Operations Research. 2009.36(12): 3215-3223.

[8] Ai, T.J., Kachitvichyanukul, V., A particle swarm optimization for the vehicle routing problem with simultaneous pickup and delivery. Computers \&Operations Research. 2009. 36(5), 1693-1702. 